\title{
A model of chronic lung allograft rejection in the rat
}

\author{
W. Jungraithmayr*,\#, P. Vogt ${ }^{\dagger}$, I. Inci*, S. Hillinger*, S. Arni*, S. Korom and W. Weder*
}

ABSTRACT: Bronchiolitis obliterans, the pathological hallmark of chronic pulmonary rejection, severely impacts long-term survival following lung transplantation. However, experimental reproduction of this pathophysiological phenomenon has not been achieved with contemporary in vivo models. Here, a model of chronic rejection is described, with sensitised recipients receiving unilateral orthotopic rat lung transplants.

Lewis rats, sensitised with skin from brown Norway rats 7 days before receiving left lung transplants from donors that were Lewis $\times$ brown Norway $F_{1}$ hybrids, were analysed during day 21-84. The development of chronic rejection was modulated by a treatment with rapamycin and cyclosporin, and characterised histologically, immunohistochemically and by reverse transcriptase PCR.

Characteristic histopathological changes leading to chronic rejection were induced over time by an initial treatment with cyclosporin in the presence of continuous rapamycin application. At day 84, fibrotic lesions replaced the respiratory epithelium within small bronchioles, with strong expression of smooth muscle $\alpha$-actin and upregulation of mRNA for T-helper cell type-1 cytokines, smooth muscle $\alpha$-actin, transforming growth factor- $\beta$ and CC chemokine ligand 5, but decreased forkhead box protein P3 gene expression.

A reproducible and clinically relevant experimental set-up for progressive chronic rejection in rat pulmonary allografts is described. This model will permit better understanding of the pathological changes of small airways during the development of bronchiolitis obliterans, and may serve as an in vivo set-up for testing the efficacy of novel therapeutic interventions.

KEYWORDS: Bronchiolitis obliterans, chronic rejection, orthotopic rat lung transplantation, rapamycin, sensitisation

urgical technique and anti-infective and immunosuppressive therapy have increased overall 1-yr survival following lung transplantation (Tx) to 85-90\% [1], but longterm prognosis has not improved similarly. The major obstacle in the way of unlimited graft acceptance remains the phenomenon of chronic rejection (CR). Several aetiological factors, both immunological and nonimmunological, have been linked with CR. Donor-associated parameters, preservation and reperfusion injury, viral infection and acute rejection (AR) episodes influence the development of CR [2, 3]. In contrast to AR, where the histological pattern reveals perivascular lymphocytic infiltrates and parenchymal damage, biopsy specimens from pulmonary grafts undergoing CR show proliferative remodelling within small airways, leading to luminal obstruction and organ-specific lesions that are described as bronchiolitis obliterans (BO). BO may be found in $30-60 \%$ of recipients within the first 3 yrs following $\mathrm{Tx}$, and corresponds to fibro-obliterative changes of small bronchioles, the hallmark of CR [4].

Several experimental approaches have been developed to simulate $\mathrm{CR}$ in vivo, since a reproducible animal model for the disease is essential. A widespread set-up employs the subcutaneous placement of tracheal rings [5]. However, these models have major drawbacks, which limit their comparability to clinical reality since they are nonphysiological. First, tracheal segments undergo severe initial ischaemia, which may alter the course of the subsequent immune response. Following this initial ischaemic phase, viability of the tracheal rings relies on diffusion from the surrounding tissue alone. Secondly, physiological ventilation as a central functional aspect in lung Tx does not occur. Thirdly, this concept does not take the anatomical and physiological differences between large and small airways into consideration, and, fourthly, the observed changes occur within a short span
AFFILIATIONS

*Division of Thoracic Surgery,

"Dept of Clinical Pathology,

University Hospital Zurich, Zurich, Switzerland.

${ }^{\#}$ Dept of Thoracic Surgery, University Hospital Freiburg, Freiburg,

Germany.

CORRESPONDENCE

W. Jungraithmayr

Division of Thoracic Surgery

University Hospital Zurich

Raemistr. 100

8091 Zurich

Switzerland

E-mail: wolfgang.jungraithmayr@

usz.ch

Received:

July 142009

Accepted after revision:

Sept 152009

First published online:

Oct 192009 
of time, in contrast to the slowly developing BO in human lung transplant recipients. Other approaches include end-to-end orthotopic tracheal Tx [6] or direct insertion (heterotopic) of an allograft trachea into the recipient's pulmonary parenchyma [7]. However, these models continued to suffer disadvantages similar to the heterotopic tracheal Tx technique. Attempts to create $\mathrm{CR}$ in orthotopic lung Tx models in the rat have also been unrewarding $[8,9]$.

Recipient sensitisation towards major histocompatibility complex (MHC) antigens due to multiple blood transfusions and previous engraftments and pregnancies constitutes a significant problem in current Tx medicine. These patients are at a greater risk of AR episodes, which, even if successfully treated, may predispose to a progressive decline in organ function over the years in comparison to unsensitised hosts. In 1994, KOROM et al. [10] developed a model of CR in heterotopically transplanted rat cardiac allografts. Simulating recipient sensitisation, they chose an established model of accelerated rejection in combination with rapamycin (RPM) therapy [10]. Employing this inhibitor of mammalian target of RPM [11] markedly diminished AR and accelerated rejection episodes, but did not prevent the progressive loss of specific organ function that occurred while the morphological pattern of $C R$ developed [12].

Drawing from experience in this experimental set-up, a novel approach of inducing CR in a rat orthotopic lung Tx model in sensitised recipients is described here.

\section{MATERIALS AND METHODS}

\section{Experimental model}

Inbred male rats (Harlan, Venray, the Netherlands) weighing 250-300 g were used. Animals received adequate care according to The Principles of Laboratory Animal Care (promulgated in 1985 and most recently revised in 1996 [13]), and the study was approved by the local animal committee (Licence No. 217/ 2006). For allogeneic Tx $(n=5-8)$, left pulmonary grafts were recovered (Lewis $(\mathrm{LEW}) \times$ brown Norway $(\mathrm{BN}) \mathrm{F}_{1}$ hybrids) and transplanted into LEW recipients applying orthotopic left single-lung Tx, using a cuff technique [14]. For syngeneic Tx $(n=4)$, pulmonary grafts from LEW rats were used. For presensitisation, a skin flap of $3 \times 3 \mathrm{~cm}$ was orthotopically placed on the dorsal thorax of prospective recipients at day -7 . In order to identify the optimal therapeutic regimen of RPM application, a series of pilot experiments were performed, assessing RPM doses of $0.25-0.75 \mathrm{mg} \cdot \mathrm{kg}^{-1} \cdot \mathrm{day}^{-1}$ and cyclosporin A (CsA) doses of $2.5 \mathrm{mg} \cdot \mathrm{kg}^{-1} \cdot$ day $^{-1}$ over various time frames (fig. 1).

\section{Histological assessment}

For morphometric examination of the sections, standard staining with haematoxylin and eosin was used. All grafts were evaluated according to the classification of the working formulation for classification and grading of pulmonary rejection (International Society for Heart and Lung Transplantation (ISHLT) grading).

\section{Immunohistochemistry for CD68, CD3 and smooth muscle $\alpha$-actin}

Immunohistochemistry was performed according to a previously established protocol [15]. The primary antibody for
CD68 and CD3 (AbD Serotec, Düsseldorf, Germany) were applied at dilution of 1:200, and for smooth muscle $\alpha$-actin ( $\alpha$ SMA) (Sigma-Aldrich, St Louis, MO, USA) at a dilution of 1:100, incubated for $2.5 \mathrm{~h}$, washed in Histo-Tris ( $5 \mathrm{mM}$ Tris, $154 \mathrm{mM} \mathrm{NaCL}, \mathrm{pH} 7.5$ ), and incubated for $30 \mathrm{~min}$ with the biotinylated secondary link antibody (Vector Laboratories, Servion, Switzerland) before being washed twice more in Histo-Tris. They were then incubated for $30 \mathrm{~min}$ at room temperature with avidin-biotin complex, and the signal was detected with 3,3'-diaminobenzidine tetrahydrochloride (DAB), producing a brown stain at the site of the reaction. Sections were lightly counterstained with Mayer's haematoxylin. Haematoxylin-eosin counterstaining of corresponding sections identified mononuclear cells as alveolar macrophages (AMs). Three different representative sections from each graft were analysed. The AMs and T-cells in 20 alveoli were counted in a blinded fashion.

\section{Immunofluorescence staining for T-helper cell type-1 and -2 cytokines}

Lung tissue was fixed with optimum cutting temperature (OCT) mounting medium, immediately frozen in liquid nitrogen and stored at $-80^{\circ} \mathrm{C}$ until use. The tissue was cut into $5-\mu \mathrm{m}$ slices at $-22^{\circ} \mathrm{C}$ and put on to positively charged glass slides and stored at $-80^{\circ} \mathrm{C}$ until use. The slides were washed in 2:1 methanol/acetone for $10 \mathrm{~min}$ and air dried. After washing in PBS, sections were blocked for $1 \mathrm{~h}$ in $1 \%$ bovine serum albumin (BSA) in PBS containing 0.05\% Tween 20 (SigmaAldrich) and goat serum at a dilution of 1:20. After incubation with the first mouse anti-rat antibody (interleukin (IL)-2 and -12 1:50; IL-4 and -10 1:200), slides were washed three times for $15 \mathrm{~min}$ and incubated with fluorescein-isothiocyanate-labelled goat anti-mouse immunoglobulin $\mathrm{G}$ at a dilution of 1:100 (AbD Serotec) in 1\% BSA in PBS, 0.05\% Tween 20 for $1 \mathrm{~h}$. After an additional rinse, counterstaining of the cell nucleus was performed with 4,6-diamino-2-phenylindole (1:1,000 in PBS) for $5 \mathrm{~min}$, and slides were mounted using Dako Fluorescence Mounting Medium (Dako, Carpinteria, CA, USA). Staining was analysed with a Leica AF6000 fluorescent microscope (Leica Microsystems, Wetzlar, Germany). Three different representative sections from each graft were analysed. The positively stained cells in 20 alveoli were counted in a blinded fashion.

\section{Quantitative RT PCR for T-helper cell type-1 and -2 cytokines, transforming growth factor- $\beta$, RANTES, $\alpha-S M A$ and forkhead box protein P3}

Total RNA was extracted from $50 \mathrm{mg}$ lung tissue using TRIzol reagent (Invitrogen, Paisley, UK). A total of $5 \mu \mathrm{g}$ RNA were reverse transcribed using the Thermoscript RT-PCR kit (Invitrogen, Basle, Switzerland), yielding the cDNA template. Quantitative real-time PCR amplification and data analysis were performed using an ABI-Prism 7000 Sequence Detection System. TaqMan gene expression assays (PE Applied Biosystems, Rotkreuz, Switzerland) for IL-2 (assay identifier Rn99999181_m1), IL-12 (Rn00575112_m1), interferon (IFN)- $\gamma$ (Rn00594078_m1), IL-10 (Rn00563409_m1), IL-13 (Rn00587615_ m1), transforming growth factor (TGF)- $\beta$ (Rn99999016_m1), $\alpha$-SMA (Rn01759928_m1), regulated on activation, normal T-cell expressed and secreted (RANTES) (Rn00579590_m1) and forkhead box protein P3 (FoxP3) (Rn01525085_m1) were 
a)
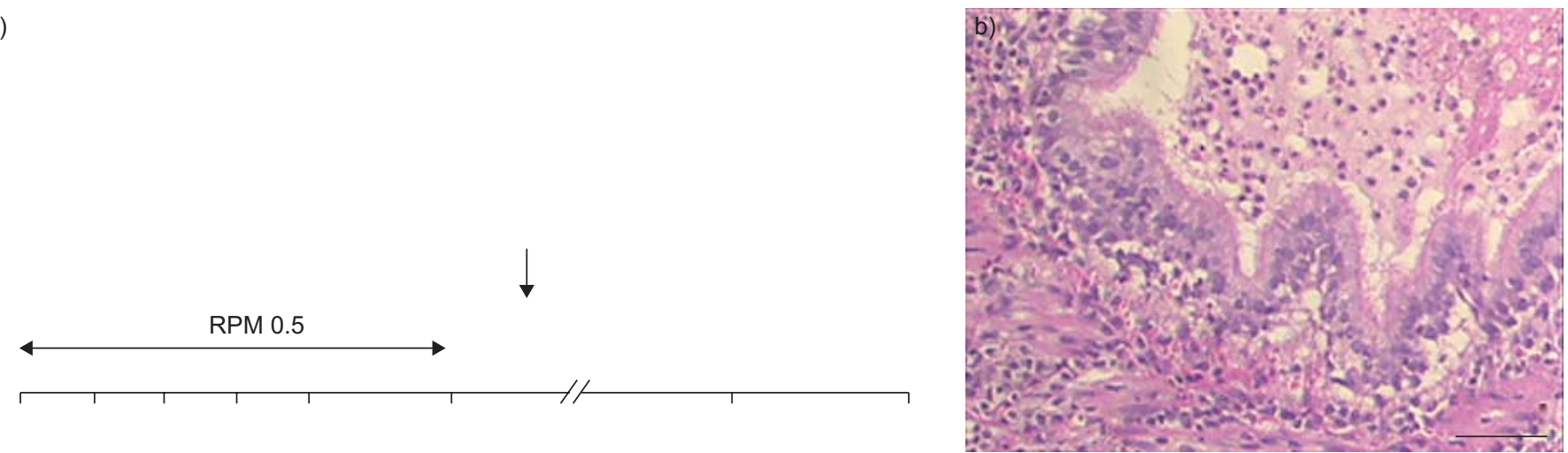

c)
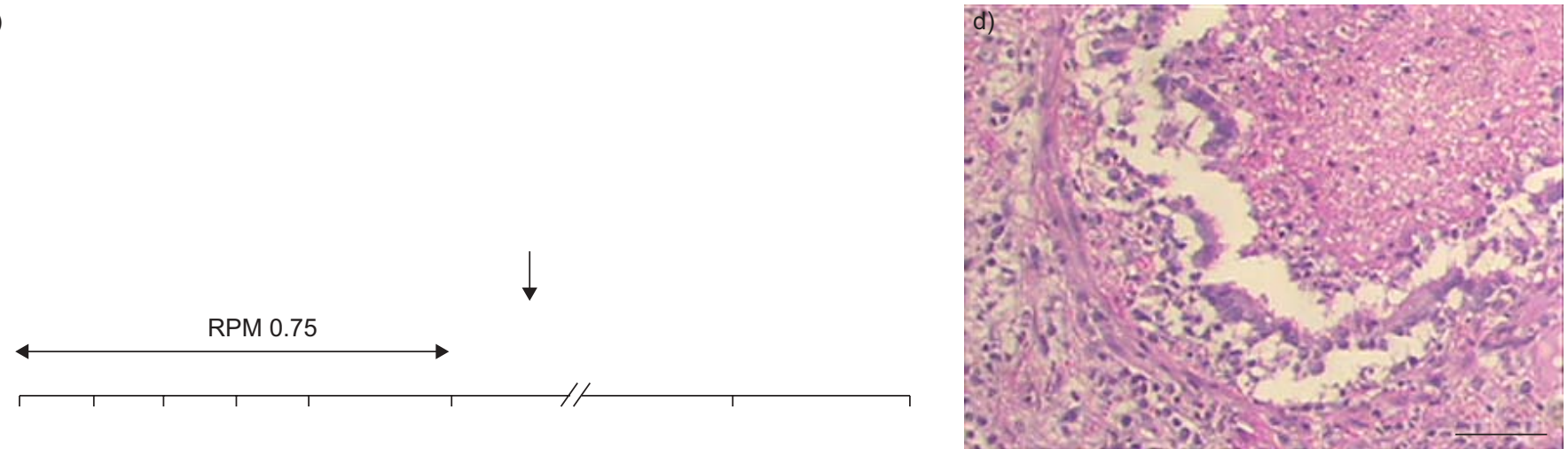

e)
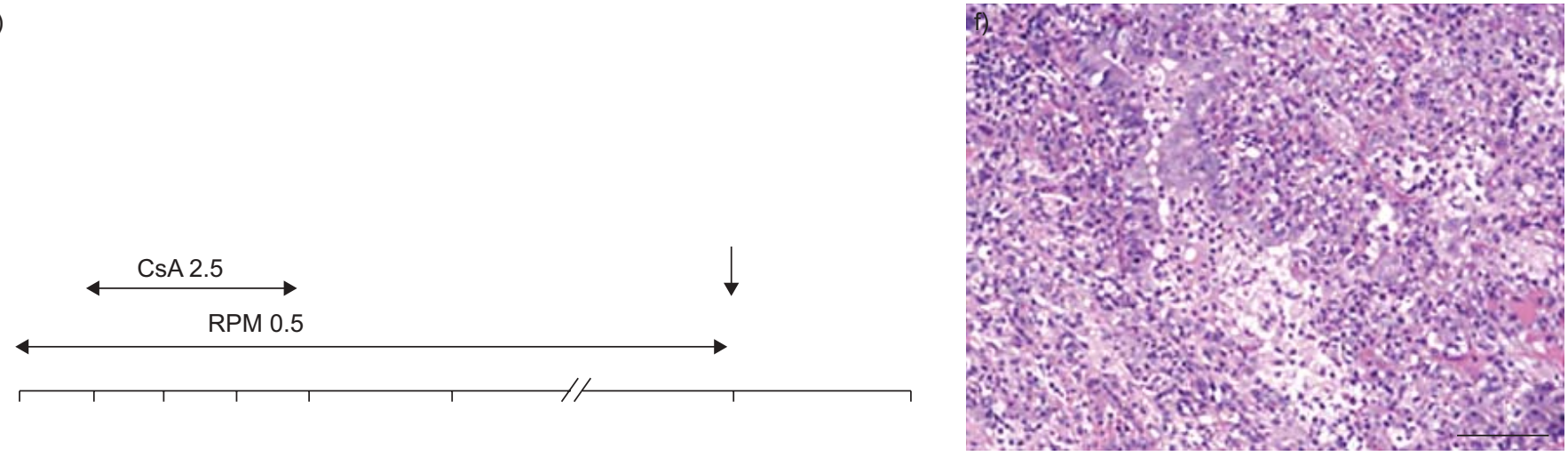

g)

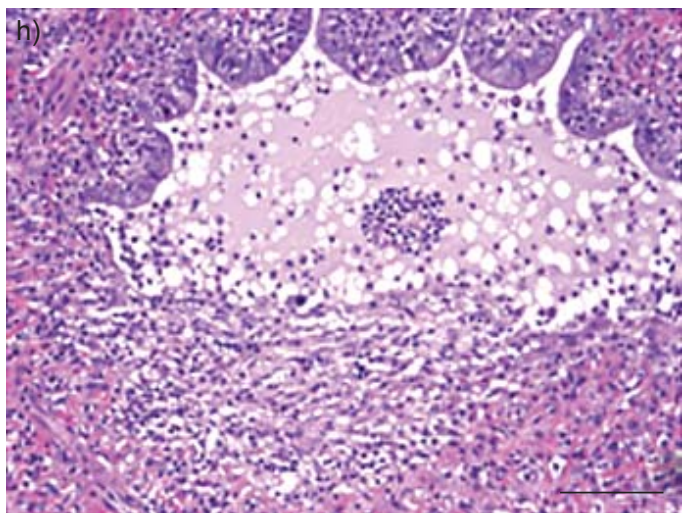

FIGURE 1. a, c, e, g) Time course of various treatments with rapamycin (RPM) and cyclosporin A (CsA) (doses shown are in milligrams per kilogram per day). -------: 3 doses.week ${ }^{-1}$. Vertical arrows indicate day of harvest. Histology, in paraffin sections, of corresponding grafts harvested following allogeneic transplantation from Lewis $(L E W) \times$ brown Norway $F_{1}$ hybrids to sensitised LEW rats on: b) day 42; d) day 42; f) day 70; and h) day 84 (haematoxylin-eosin stain). The fibroproliferative changes displayed in $\mathrm{h}$ ) occurred in $80 \%$ of animals, and were found in $80 \%$ of all bronchioles in whole-lung specimens in this group ( $n=5-8$ per group). b, d) Scale bars $=50 \mu \mathrm{m}$. $\mathrm{f}$, h) Scale bars $=100 \mu \mathrm{m}$ 
used to quantify the mRNA expression of the respective genes. The mRNA expression levels for each sample were normalised to that of 18S RNA. The results obtained are presented as the fold induction versus baseline levels in control syngeneic transplanted rats.

\section{Statistical analysis}

Data analysis was performed using SPSS for Windows 15.0 (SPSS, Inc., Chicago, IL, USA). All data are expressed as median (interquartile range). For categorical values, Pearson's Chi-squared test was used. The Mann-Whitney test was performed to compare the differences between the two groups. A p-value of $<0.05$ was considered significant.

\section{RESULTS}

\section{Histological changes in sensitised allografts during $\mathbf{C R}$}

Recipients were treated with various RPM doses at different time-points (fig. 1). Allografts were analysed 42 days after Tx, under the lowest dose of RPM $\left(0.25 \mathrm{mg} \cdot \mathrm{kg}^{-1} \cdot \mathrm{day}^{-1}\right)$, and showed near-complete necrosis due to AR. When increasing the dose of $\mathrm{RPM}$ to $0.5 \mathrm{mg} \cdot \mathrm{kg}^{-1} \cdot \mathrm{day}^{-1}$, destruction and detachment of the respiratory epithelium was observed in small bronchioles (fig. 1b), with partial arterial occlusion (ISHLT grade A2-A3). When introducing $0.75 \mathrm{mg} \cdot \mathrm{kg}^{-1} \cdot \mathrm{day}^{-1} \mathrm{RPM}$, severe lymphocytic bronchiolitis and detachment of the respiratory epithelium were induced (fig. 1d), but even this high dose of RPM did not succeed in controlling the AR and vascular rejection patterns prevailed (ISHLT grade A2-A4). CsA was then introduced in order to target the acute vascular rejection phase. Introduction of low-dose CsA $\left(2.5 \mathrm{mg} \cdot \mathrm{kg}^{-1} \cdot \mathrm{day}^{-1}\right)$ during the first 3 weeks following Tx, together with RPM $\left(0.5 \mathrm{mg} \cdot \mathrm{kg}^{-1} \cdot \mathrm{day}^{-1}\right)$, effectively suppressed the acute vascular rejection phase and resulted in striking graft preservation up to 70 days after Tx; however, periand transbronchial lymphocytic sequestration occurred more frequently and more severely (fig. 1f). Reducing the CsA treatment phase to 1 week following engraftment and with continuous application of $0.5 \mathrm{mg} \cdot \mathrm{kg}^{-1} \cdot \mathrm{day}^{-1} \mathrm{RPM}$, substantial destruction of respiratory bronchial epithelium was observed, with development of fibroproliferation, partially replacing the respiratory epithelium 84 days after Tx (fig. 1h). At this timepoint, there was strong positivity for $\alpha$-SMA within fibrotic lesions (fig. 2).

\section{Histology in sensitised syngrafts}

In order to investigate solely the effect of skin sensitisation, syngeneic LEW rats that had received $\mathrm{BN}$ skin 7 days before Tx were used. Histology on day 42 after Tx revealed mild thickening of the alveolar wall, with low leukocytes number (fig. 3a) and without oedema formation or inflammation of bronchioles (fig. 3b). On day 60 after Tx, syngeneic grafts displayed moderate alveolar wall thickening, accompanied by a mild sequestration of AMs and neutrophils into the alveolar wall (fig. 3c). The peribronchial space and, to a lesser extent, the perivascular space were discretely enlarged, and filled with AMs, whereas bronchioles were surrounded by a cuff of lymphocytes (fig. 3d). There was no evidence of intra-alveolar oedema formation.

\section{Macrophages and T-cells in allografts and syngrafts}

The activation of AMs and T-cells plays a critical role in the initiation of the pathways leading to CR. On day 21, AMs, and also CD3-positive T-cells, were the most prominent cells within allografts ( $n=6$ per group) (fig. 4$)$.

\section{T-helper cell type-1 and -2 cytokines in allografts}

T-helper cell (Th) type-1-related cytokines are considered to be dominant over Th2 cytokines during AR episodes. The Th1 cytokines IL-2 and -12 were detected with increased intensity at day 21 after Tx (fig. 5a-d), whereas the Th2 cytokines IL-4 and -10 were expressed to a lesser extent within allografts $(n=6$ per group) (fig. $5 \mathrm{e}-\mathrm{h})$.

\section{mRNA expression of Th1 and Th2 cytokines, TGF- $\beta$, RANTES, $\alpha$-SMA and FoxP3 during chronic rejection}

Quantitative real-time PCR analysis performed in allografts relative to syngrafts demonstrated greater expression of mRNA at day 21 after Tx for IL-2, IL-12 and IFN- $\gamma$, and at day 84 for IL-2, IL-12, IFN- $\gamma$, IL-10, IL-13, TGF- $\beta, \alpha$-SMA and RANTES, but, at day 84, a decreased expression difference between sensitised allografts and syngrafts for FoxP3 ( $n=4$ per group for all) (fig. 6).

\section{DISCUSSION}

Based on an established cardiac CR model, a clinically relevant experimental set-up with stable reproducibility for the studying of CR in sensitised rat recipients is described here. RPM, in combination with CsA, in a defined treatment algorithm led to the progressive development of the characteristic fibroproliferative changes associated with $\mathrm{BO}$ in orthotopically transplanted pulmonary allografts.

The murine heterotopic tracheal Tx model has been extensively used for the study of BO [16]. Although this model is technically easy to perform, it is associated with major limitations. Alternative models have utilised orthotopic tracheal Tx [6] or intraparenchymal insertion of allogeneic tracheal segments into the host lung [7]. However, these approaches are hampered by similar pitfalls. To date, experimental in vivo models investigating small airway changes associated with BO in perfused organ Tx yield heterogeneous results and lack reproducibility: Tx models of moderately histo-incompatible inbred rat strain combinations (between F344 and Wistar Kyoto animals) revealed interstitial and peribronchiolar fibrosis but no pathological changes within small airways [8]. Late airway changes were detected 6 months after Tx by LEE et al. [9], but the lesions were expressed infrequently. A first-generation rat model, between LEW and $\mathrm{BN}$, was chosen with the intention of attenuating AR episodes by the fully MHC-II mismatched strains while maintaining a sufficient immunogeneic background. However, in this strain combination, AR episodes occluded arteries and rendered allografts nonfunctional. This was particularly surprising since RPM therapy has been shown to reverse AR [17]. In contrast to various engrafted solid organs, where interaction between host and recipient are confined to the vasculature of the transplant, the lung has a more complex anatomy. Bronchial and pulmonary circulation plus the alveolar exchange surface constitute a vast area for interaction between the recipient and the milieu extérieur, which could, in part, explain the marked rejection early during the course of $C R$.

RPM therapy markedly diminishes AR and accelerated rejection episodes, but it does not prevent the progressive loss 

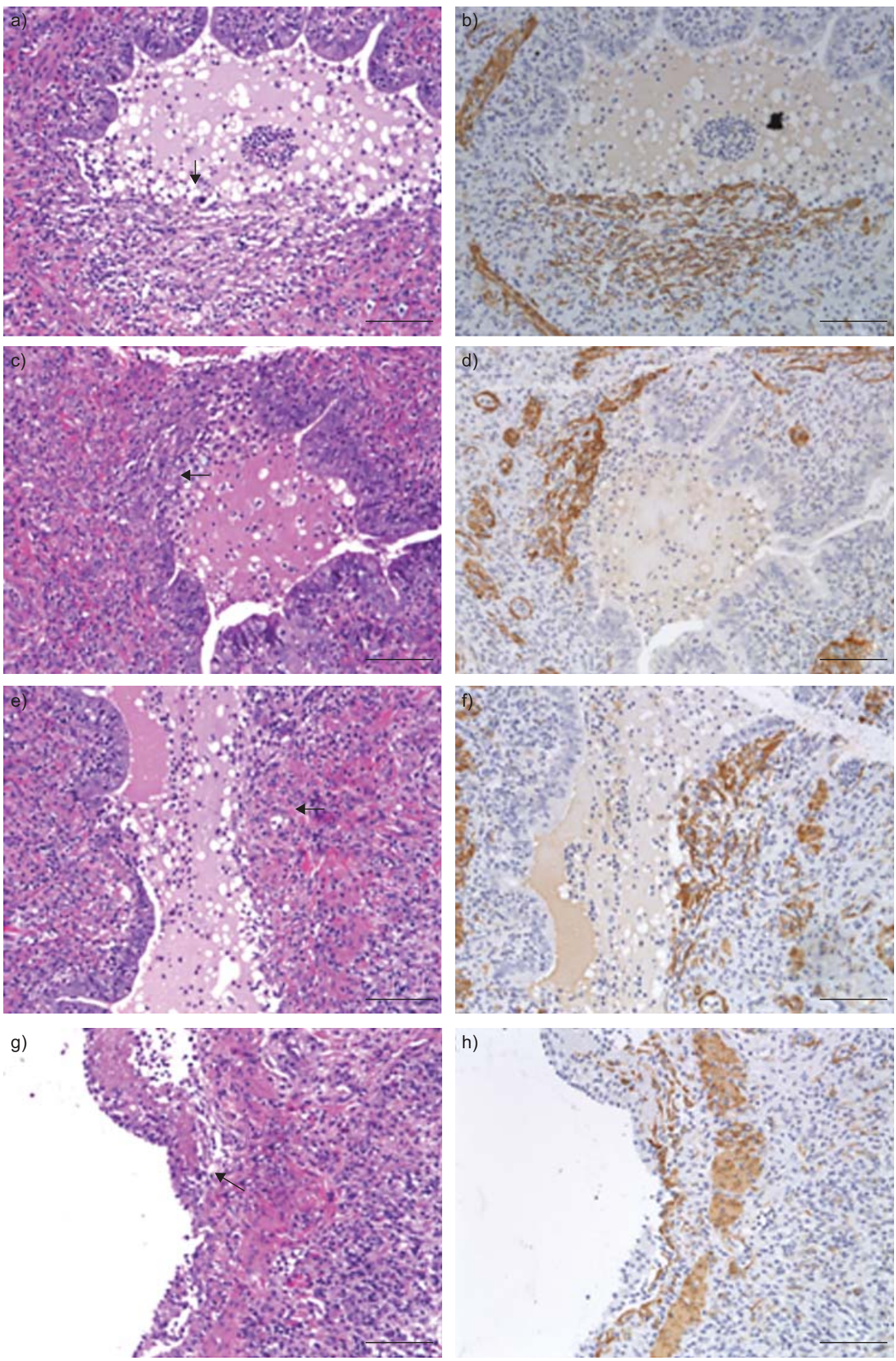

FIGURE 2. Haematoxylin-eosin staining ( $a, c, e$ and g) and immunohistochemistry for smooth muscle $\alpha$-actin $\left(\alpha\right.$-SMA) (b, $d$, f and h) in Lewis (LEW) $\times$ brown Norway $F_{1}$ hybrid lung allografts, transplanted into sensitised LEW recipients, 84 days after transplantation $(n=8)$. The images are randomly chosen representative sections from the corresponding location. The $\alpha$-SMA primary antibody was detected using a horseradish-peroxidase-coupled antibody and 3,3'-diaminobenzidine tetrahydrochloride, with brown staining of myofibroblasts within bronchioles. Arrows indicate the site of loss of respiratory epithelium and the development of fibroproliferative changes. Scale bars $=100 \mu \mathrm{m}$. 

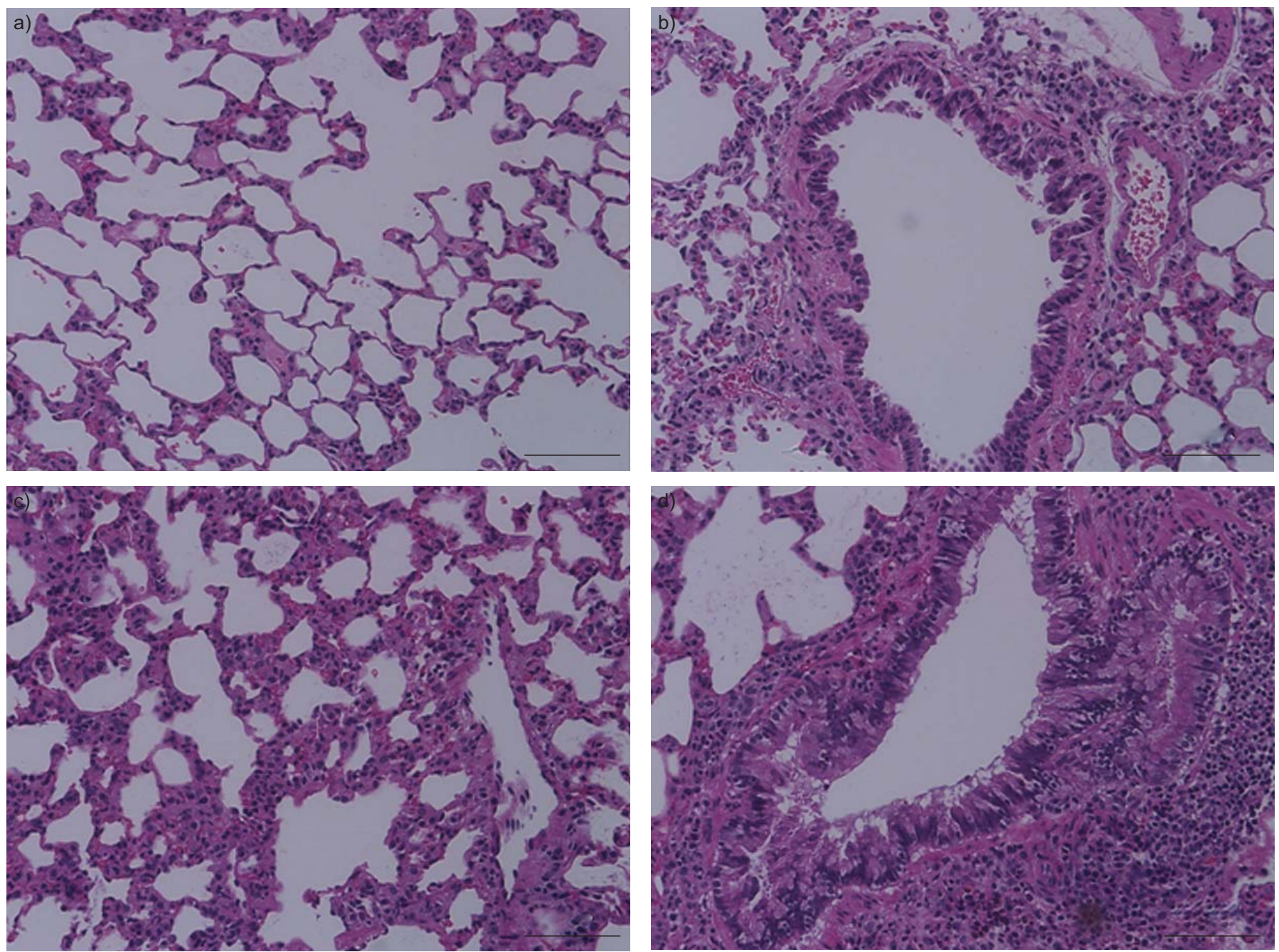

FIGURE 3. Alveolar and bronchial histology, respectively, of syngeneic grafts (Lewis to Lewis; $h=4$ ) in previously sensitised recipients: $a$, b) 42 days and c, $d) 60$ days after transplantation (haematoxylin-eosin staining.). The ultrastructure of syngrafts was preserved at day 42 , whereas syngeneic grafts showed minimal signs of alveolar and peribronchial inflammation at day 60 . Scale bars $=100 \mu \mathrm{m}$.

of specific organ function. Interestingly, RPM does not effectively abrogate the late expression of pro-inflammatory macrophage-associated gene transcripts, such as RANTES, monocyte chemotactic protein-1 and IL-12 [12]. When looking for the presence of activated AMs, a significant accumulation of AMs was found in allografts early during CR. However, levels of RANTES, which plays a pivotal role in recruiting mononuclear phagocytes during the pathogenesis of $\mathrm{BO}$ [18], were also found to be elevated at both early and late timepoints within the time-frame of analysis. Furthermore, TGF- $\beta$ mRNA levels were described as being higher in RPM-treated animals during the $\mathrm{CR}$ process compared to untreated recipients [10]. Confirmation of increased levels of TGF- $\beta$ at day 84 rendered this mediator relevant during the development of CR.

Implementing low-dose CsA in the treatment regimen allowed for a shift towards bronchial immune interaction during the course of rejection. The characteristic stages of destruction of small airways, which were all in accordance with the description of the working formulation in the diagnosis of lung rejection, could then be observed [19]. The recruitment of myofibroblasts, which partially replaced the respiratory epithelium at day 84 , was shown to be an inevitable condition for fibrous obliteration in heterotopic tracheal allografts [20]. The significance of this histological feature is increasingly recognised by others [21], and current literature provides evidence that the myofibroblast/ $\alpha$-SMA phenotype can be a result of the upregulation of TGF- $\beta$ trough activation of various pathways, such as the mitogen-activated protein kinase and c-Jun pathway [22].

Individuals who have received transplants and who have been exposed to multiple blood transfusions, previous engraftments and pregnancies are thought to be sensitised towards MHC antigens, and are, therefore, at a greater risk of AR episodes, which, even if successfully treated, predispose to a chronic decline in organ function over the years in comparison to unsensitised hosts. In the heterotopic heart model, presensitisation in combination with RPM resulted in characteristic signs of $\mathrm{CR}$, with the morphological picture of accelerated graft arteriosclerosis [23]. However, in this model of CR, 

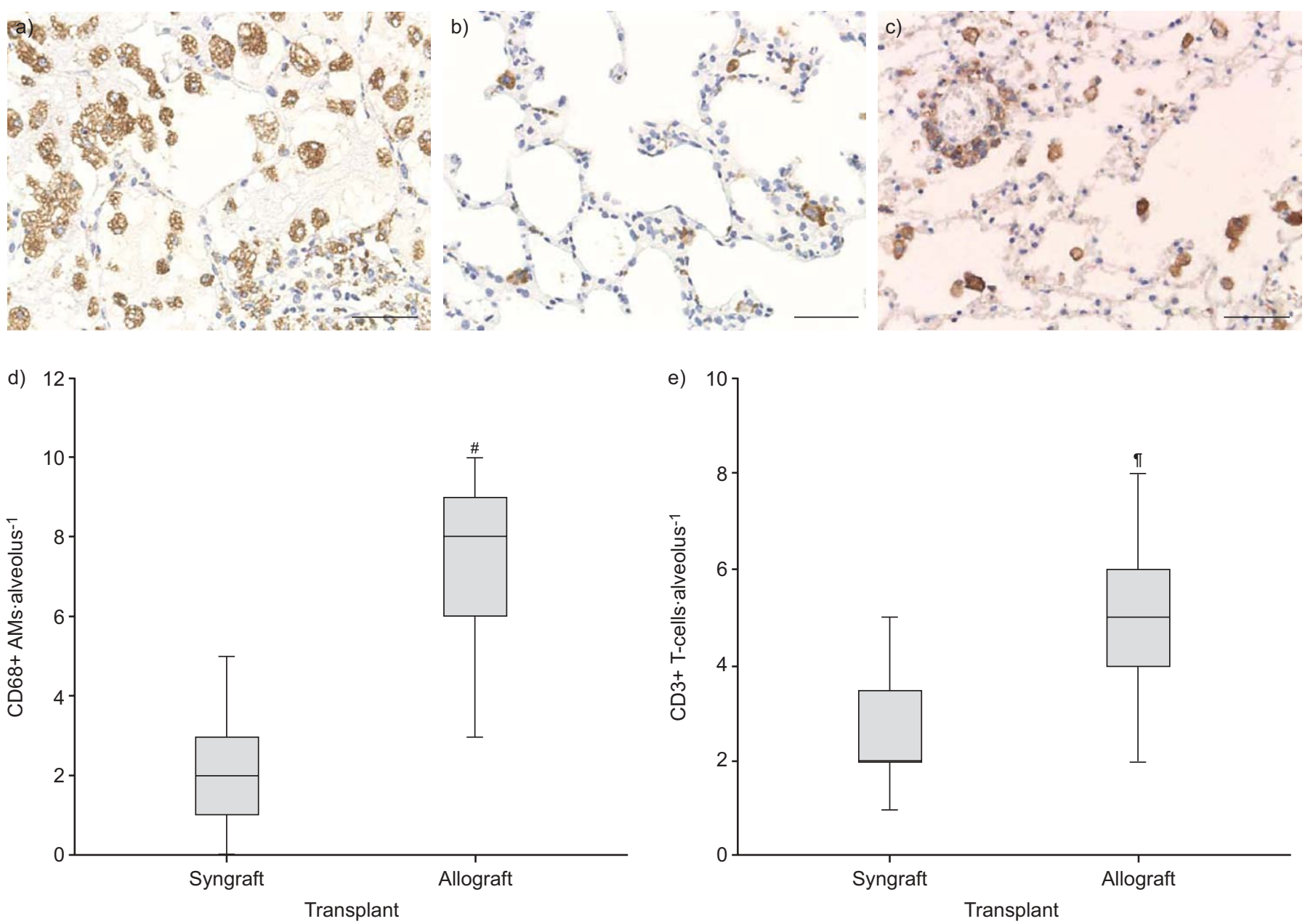

FIGURE 4. CD68-positive activated alveolar macrophages (AMs) 21 days after: a) allograft transplantation (Tx) of Lewis (LEW) $\times$ brown Norway $F_{1}$ hybrid (LBNF 1 ) to sensitised LEW; and b) syngeneic Tx (LEW to LEW). CD68-positivity was sparse in the latter. c) Perivascular lymphocytic cuff of CD3-positive T-cells 21 days after allograft Tx of $\mathrm{LBNF}_{1}$ to sensitised LEW. Nuclei were counterstained with haematoxylin. Scale bars $=50 \mu \mathrm{m}$. d) For CD68-positive AMs, the number of positively stained cells per alveolus was increased in sensitised allografts versus syngrafts. e) For CD3-positive T-cells, the number of cells per alveolus differed significantly between sensitised allografts and syngrafts. Boxes represent median and interquartile range; vertical bars represent the 10 th and 90 th percentiles. ${ }^{*}: p=0.0001 ; "$ : $p=0.01$ versus syngraft ( $n=6$ per group).

pre-sensitisation did not have a severe impact on CR changes. This could be due to the different immunological environment of the pulmonary tissue, or, alternatively, RPM could be inducing an attenuation of the alloreactive antibody response.

Although RAMIREZ et al. [20] did not show an effector function of alloreactive cells, of which they identified only few in BO lesions, it may be assumed that airway wound repair is dependent upon a delicate balance between pro- and antiinflammatory cytokines. Changes in this balance can influence allograft airway remodelling. Low levels of IL-12 were demonstrated to predict the development of $\mathrm{BO}$ syndrome in humans [24], whereas the domination of the Th2 response was favoured in promoting CR [25]. In a rodent heterotopic tracheal Tx model, NEURINGER et al. [26] suggested both pathways of Th to play a role during the development of CR, whereas others provided dichotomous findings regarding the role of Th1/Th2 $[9,27]$. The marked infiltration of AMs and perivascular CD3-positive T-cells most probably lead to a strong Th1 response in allografts in the present model. As has been shown in the cardiac heterotopic Tx model [12], the presence of the Th1 cytokine IL-12 in long-term allografts following RPM therapy suggests a late activation of AMs that may also contribute to the CR of pulmonary transplants.

The current literature supports a protective effect, conveyed by regulatory $\mathrm{T}$-cells, in preventing rejection [28]. We were, therefore, not surprised to observe reduced levels of FoxP3 gene expression in fibrotic allografts, although the phenomenon of BO did not affect the full circumference of bronchioles at the end of the present investigation period.

Although clear evidence for CR could be provided in small airways in physiological orthotopic allografts, some limitations of the present experimental set-up should be considered. BO did not occlude the lumen completely, possibly due to the early and pronounced effect of CsA. Choosing an even longer experimental time-frame could potentially induce a more pronounced histological picture of BO. Secondly, the role of allo-antibodies, particularly antibodies directed against recipient MHC, which are capable of damaging allograft airway epithelium, needs to be investigated since sensitisation is 

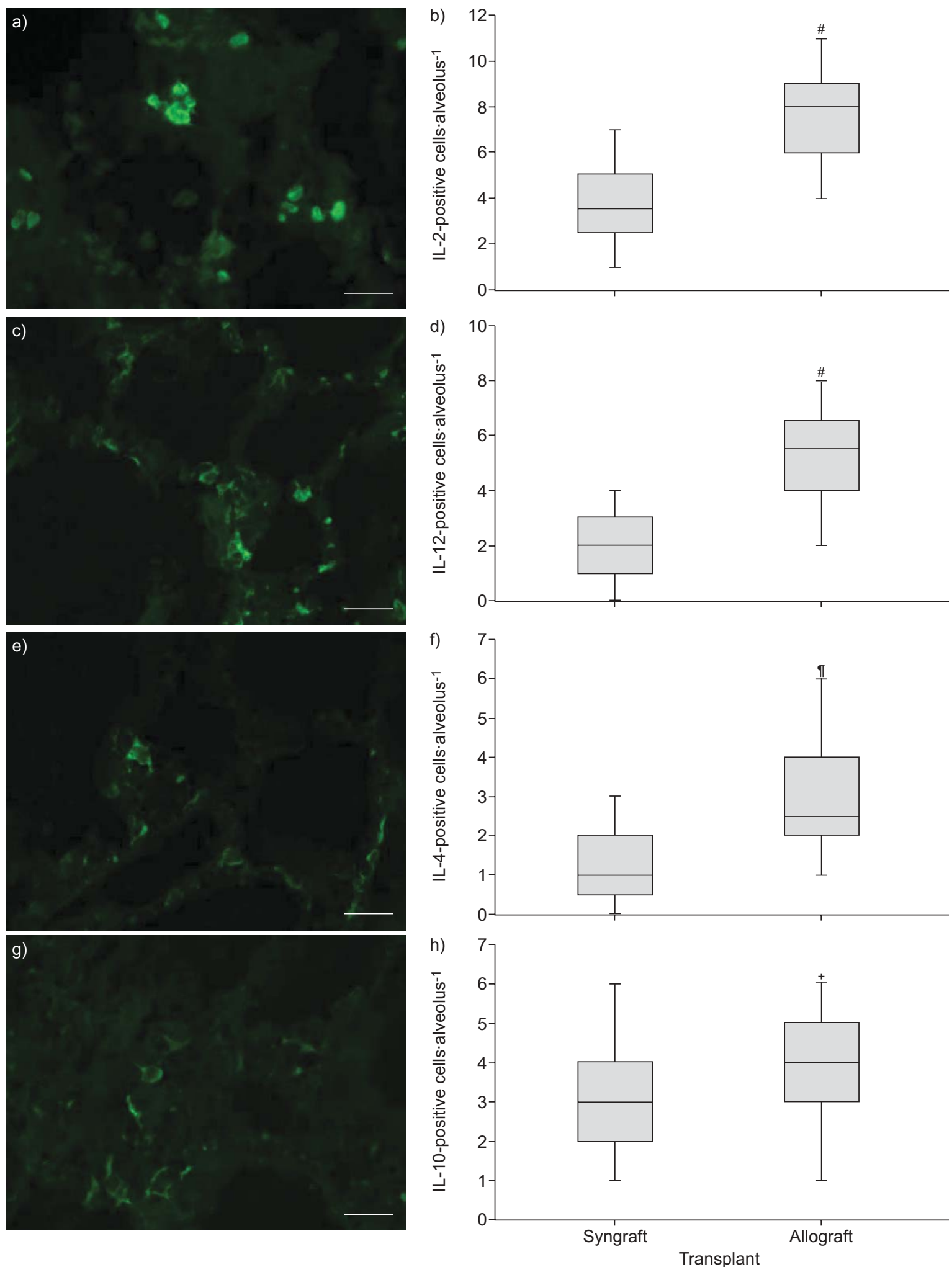

FIGURE 5. Immunofluorescence of T-helper cell type-1 and type-2 cytokines 21 days after allogeneic transplantation of Lewis (LEW) $\times$ brown Norway $F_{1}$ hybrid to sensitised LEW versus syngrafts. The cytoplasm of cytokine-secreting cells is stained green (fluorescein isothiocyanate labelling). The cytokine-secreting cells are located within the alveolar wall, partially accumulating as groups of cells around vessels and small bronchi. The alveolar structure of the pulmonary tissue appears autofluorescent in the background. There was significantly increased expression of: a) interleukin (IL)-2; and c) IL-12; and weak expression of: e) IL-4; and g) IL-10. Scale bars=20 $\mu$ m. b, d, f, h) The number of positively stained cells per alveolus was increased in sensitised allografts versus syngrafts. Boxes represent median and interquartile range; vertical bars represent the 10th and 90th percentiles. ${ }^{*}: p=0.0001 ; ": p=0.001 ;{ }^{*}: p<0.2$ (nonsignificant) versus syngraft ( $n=6$ per group). 

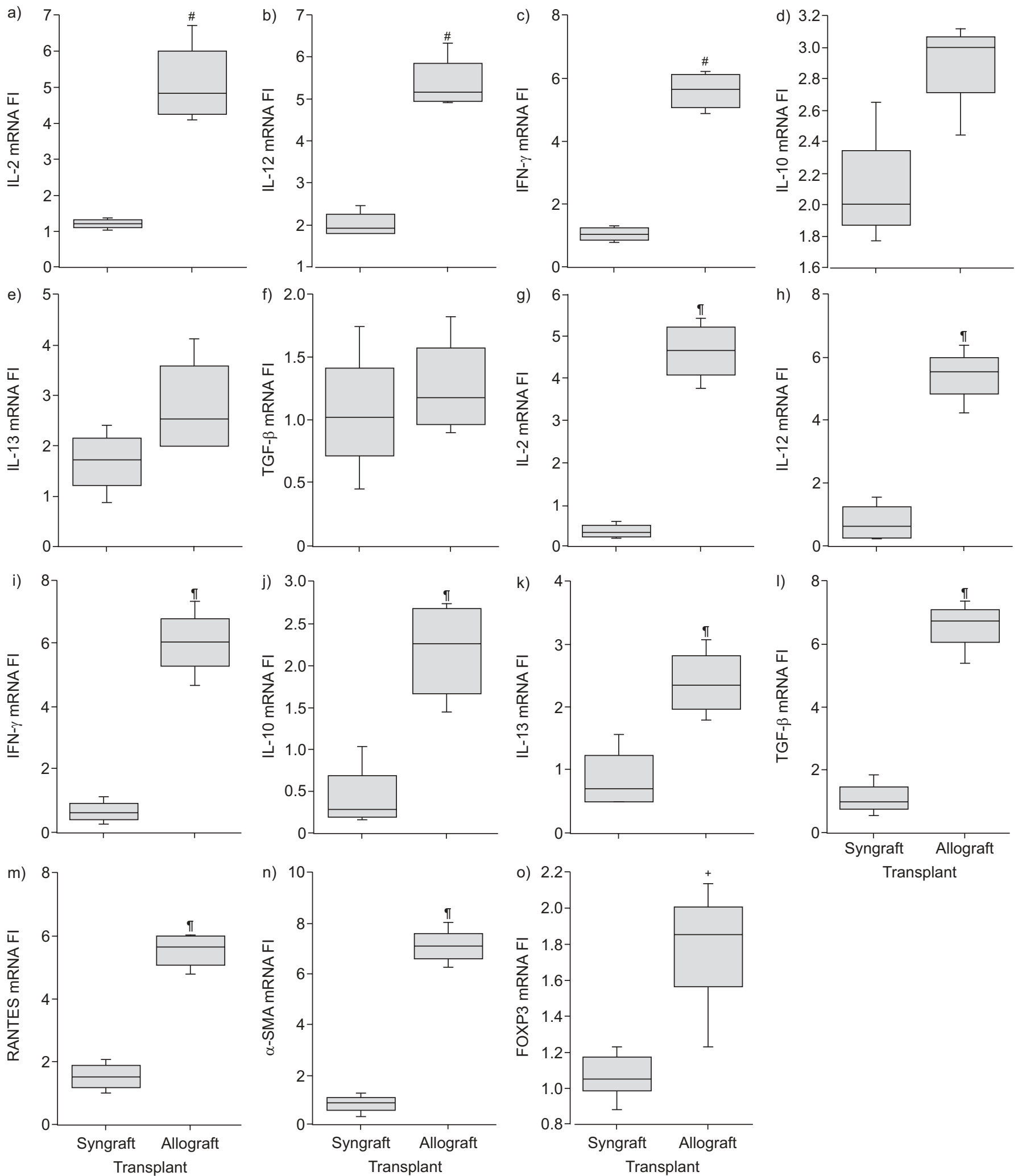

Transplant

FIGURE 6. Real-time PCR analysis in pulmonary allografts, on: a-f) day 21; and g-o) day 84 after transplantation, of mRNA expression: a, g) interleukin (IL)-2; b, h) IL-12; c, i) interferon (IFN)- $\gamma$; d, j) IL-10; e, k) IL-13; f, I) transforming growth factor (TGF)- $\beta$; m) RANTES (regulated on activation, normal T-cell expressed and secreted); n) smooth muscle $\alpha$-actin ( $\alpha$-SMA); and o) forkhead box protein P3 (FoxP3). Boxes represent median and interquartile range; vertical bars represent the 10th and 90th percentiles. Relative gene expression (mRNA) in sensitised allografts was increased in comparison with control syngrafts, but decreased for FoxP3. Fl: fold induction. ${ }^{*}$ : $p=0.003$ ๆ: $p=0.02{ }^{+}: p=0.008$ versus syngraft ( $n=4$ per group). 
known to induce the synthesis of donor antibodies that have been associated with BO. Thirdly, rat models lack the breadth of reagents and genetic manipulations available for studying both innate and adaptive immunity during CR. It was previously possible to show ventilated and perfused syngeneic grafts to be functional up to day 90 after Tx in a mouse model of orthotopic Tx [29]. This model could potentially serve as a suitable future tool for the investigation of CR.

\section{SUPPORT STATEMENT}

This work was supported by Deutsche Forschungsgemeinschaft, grant number JU 2730/1-1, Germany.

\section{STATEMENT OF INTEREST}

None declared.

\section{ACKNOWLEDGEMENTS}

We thank D. Fetz (Clinical Pharmacy, University Hospital Zurich, Zurich, Switzerland) for preparing the rapamycin solution, and $\mathrm{M}$. Bain and U. Ungethuem (both Division of Surgical Research, University Hospital Zurich) for their invaluable help with the immunohistochemistry and reverse transcriptase PCR experiments.

\section{REFERENCES}

1 Trulock EP, Christie JD, Edwards LB, et al. Registry of the International Society for Heart and Lung Transplantation: twentyfourth Official Adult Lung and Heart-Lung Transplantation Report-2007. J Heart Lung Transplant 2007; 26: 782-795.

2 Cramer DV, Qian SQ, Harnaha J, et al. Cardiac transplantation in the rat. I. The effect of histocompatibility differences on graft arteriosclerosis. Transplantation 1989; 47: 414-419.

3 Boehler A, Estenne M. Obliterative bronchiolitis after lung transplantation. Curr Opin Pulm Med 2000; 6: 133-139.

4 Valentine VG, Robbins RC, Berry GJ, et al. Actuarial survival of heart-lung and bilateral sequential lung transplant recipients with obliterative bronchiolitis. J Heart Lung Transplant 1996; 15: 371-383.

5 Hertz MI, Jessurun J, King MB, et al. Reproduction of the obliterative bronchiolitis lesion after heterotopic transplantation of mouse airways. Am J Pathol 1993; 142: 1945-1951.

6 Genden EM, Boros P, Liu J, et al. Orthotopic tracheal transplantation in the murine model. Transplantation 2002; 73: 1420-1425.

7 Sato M, Liu M, Anraku M, et al. Allograft airway fibrosis in the pulmonary milieu: a disorder of tissue remodeling. $A m \mathrm{~J}$ Transplant 2008; 8: 517-528.

8 Matsumura Y, Marchevsky A, Zuo XJ, et al. Assessment of pathological changes associated with chronic allograft rejection and tolerance in two experimental models of rat lung transplantation. Transplantation 1995; 59: 1509-1517.

9 Lee AG, Wagner FM, Giaid A, et al. Immunohistochemical characterization of inflammatory and proliferative events during chronic rejection in rat lung allografts. Transplantation 1997; 64: 465-471.

10 Korom S, Hancock WW, Coito AJ, et al. Blockade of very late antigen-4 integrin binding to fibronectin in allograft recipients. II. Treatment with connecting segment- 1 peptides prevents chronic rejection by attenuating arteriosclerotic development and suppressing intragraft $\mathrm{T}$ cell and macrophage activation. Transplantation 1998; 65: 854-859.

11 Stepkowski SM, Chen H, Daloze $\mathrm{P}$, et al. Rapamycin, a potent immunosuppressive drug for vascularized heart, kidney, and small bowel transplantation in the rat. Transplantation 1991; 51: 22-26.
12 Wasowska B, Wieder KJ, Hancock WW, et al. Cytokine and alloantibody networks in long term cardiac allografts in rat recipients treated with rapamycin. J Immunol 1996; 156: 395-404.

13 National Institutes of Health. Principles of Laboratory Animal Care. Publication No. 85-23, revised 1996. Bethesda, MD, National Institutes of Health, 1996

14 Zhai W, Cardell M, De Meester I, et al. Intragraft DPP IV inhibition attenuates post-transplant pulmonary ischemia/reperfusion injury after extended ischemia. J Heart Lung Transplant 2007; 26: 174-180.

15 Graf R, Schiesser M, Lussi A, et al. Coordinate regulation of secretory stress proteins (PSP/reg, PAP I, PAP II, and PAP III) in the rat exocrine pancreas during experimental acute pancreatitis. J Surg Res 2002; 105: 136-144.

16 McDyer JF. Human and murine obliterative bronchiolitis in transplant. Proc Am Thorac Soc 2007; 4: 37-43.

17 Chen $\mathrm{H}, \mathrm{Wu}$ J, Xu D, et al. Reversal of ongoing heart, kidney, and pancreas allograft rejection and suppression of accelerated heart allograft rejection in the rat by rapamycin. Transplantation 1993; 56 : 661-666.

18 Belperio JA, Burdick MD, Keane MP, et al. The role of the CC chemokine, RANTES, in acute lung allograft rejection. J Immunol 2000; 165: 461-472.

19 Stewart S, Fishbein MC, Snell GI, et al. Revision of the 1996 working formulation for the standardization of nomenclature in the diagnosis of lung rejection. J Heart Lung Transplant 2007; 26: 1229-1242.

20 Ramirez AM, Shen Z, Ritzenthaler JD, et al. Myofibroblast transdifferentiation in obliterative bronchiolitis: TGF- $\beta$ signaling through Smad3-dependent and -independent pathways. Am J Transplant 2006; 6: 2080-2088.

21 Pedagogos E, Hewitson TD, Walker RG, et al. Myofibroblast involvement in chronic transplant rejection. Transplantation 1997; 64: 1192-1197.

22 Hashimoto S, Gon Y, Takeshita I, et al. Transforming growth Factor- $\beta 1$ induces phenotypic modulation of human lung fibroblasts to myofibroblast through a c-Jun- $\mathrm{NH}_{2}$-terminal kinasedependent pathway. Am J Respir Crit Care Med 2001; 163: 152-157.

23 Kupiec-Weglinski JW, Sablinski T, Hancock WW, et al. Modulation of accelerated rejection of cardiac allografts in sensitized rats by anti-interleukin 2 receptor monoclonal antibody and cyclosporine therapy. Transplantation 1991; 51: 300-305.

24 Meloni F, Vitulo P, Cascina A, et al. Bronchoalveolar lavage cytokine profile in a cohort of lung transplant recipients: a predictive role of interleukin-12 with respect to onset of bronchiolitis obliterans syndrome. J Heart Lung Transplant 2004; 23: 1053-1060.

25 Zhai Y, Ghobrial RM, Busuttil RW, et al. Th1 and Th2 cytokines in organ transplantation: paradigm lost? Crit Rev Immunol 1999; 19: 155-172.

26 Neuringer IP, Mannon RB, Coffman TM, et al. Immune cells in a mouse airway model of obliterative bronchiolitis. Am J Respir Cell Mol Biol 1998; 19: 379-386.

27 Sumitomo M, Sakiyama S, Tanida N, et al. Difference in cytokine production in acute and chronic rejection of rat lung allografts. Transpl Int 1996; 9: Suppl. 1, S223-S225.

28 Meloni F, Vitulo P, Bianco AM, et al. Regulatory CD4+CD25+ T cells in the peripheral blood of lung transplant recipients: correlation with transplant outcome. Transplantation 2004; 77: $762-766$.

29 Jungraithmayr WM, Korom S, Hillinger S, et al. A mouse model of orthotopic, single-lung transplantation. J Thorac Cardiovasc Surg 2009; 137: 486-491. 